\title{
The use of acoustic emissions technique in the monitoring of fracturing in concrete using soundless chemical demolition agent
}

\author{
Vassilios Saltas, Despoina Peraki \\ Laboratory of Geophysics and Seismology - UNESCO Chair in Solid Earth Physics and Geobazards Risk Reduction, \\ Technological Educational Institute of Crete, Greece \\ vsaltas@chania.teicrete.gr, bttp://orcid.org/0000-0003-4958-2876 \\ desnikper@gmail.com \\ Filippos Vallianatos \\ Laboratory of Geophysics and Seismology - UNESCO Chair in Solid Earth Physics and Geohazards Risk Reduction, \\ Technological Educational Institute of Crete, Greece \\ Dept of Geophysics-Geothermics, Faculty of Geology and Geoenvironment, National and Kapodistrian University of Athens, Greece \\ fvallian@geol.uoi.gr
}

\begin{abstract}
Soundless chemical demolition agents (SCDAs) have been used during the last decades in the demolition of boulders and concrete structures as well as in open-surface and sub-surface rock excavation, as an alternative to the use of explosives posing safety risks. However, the knowledge of the governing fracture mechanisms in brittle materials is rather limited. In the present work, we thoroughly investigate the use of the acoustic emission technique to study the SCDA-induced fracture process in concrete blocks. Energy-related features and waveform parameters of the recorded $\mathrm{AE}$ activity are correlated to the fracture mode of the concrete where a quasi-static behavior is observed. Monitoring of the progressive fracture is also achieved by the 3D localization of the AE sources. The distribution of the inter-event times of the recorded hits is further analyzed in the context of non-extensive statistical physics.
\end{abstract}

KEYwORDS. Acoustic emissions; Fracture; Soundless chemical demolition agent; Concrete; Non-extensive statistical physics; Tsallis entropy.

\section{open 6 access}

Citation: Saltas, V., Peraki, D., Vallianatos, F., The use of acoustic emissions technique in the monitoring of fracturing in concrete using soundless chemical demolition agent, Frattura ed Integrità Strutturale, 50 (2019) 505-516.

Received: 29.01.2019

Accepted: 27.05.2019 Published: 01.10.2019

Copyright: (C) 2019 This is an open access article under the terms of the CC-BY 4.0, which permits unrestricted use, distribution, and reproduction in any medium, provided the original author and source are credited.

\section{INTRODUCTION}

$\mathrm{S}$ oundless chemical demolition agents (SCDAs) have been used during the last decades in the demolition of boulders and concrete structures as well as in open-surface and sub-surface rock excavation, as an effective alternative to the use of explosives posing safety risks [1-5]. Recently, the potential application of SCDA fracking in unconventional oil and gas recovery as well as in mineral processing applications has been under investigation $[5,6]$. The effect of the non- 
explosive cracking agents on fracturing of the surrounding media inside a borehole is based on the volume thermal expansion of the cracking agent, caused by the exothermic hydration reaction of $\mathrm{CaO}$ under confined conditions. In the proper mixing ratio of the cracking agent to water, the subsequent coagulation and rigidity of the mixture results in the development of high expansive pressures within the pre-drilled holes that eventually causes a complex fracture network in the concrete or the rock mass [7].

In addition to commercial use, non-explosive expansion material has been utilized in laboratory experiments to evaluate its fracturing capacity of synthetic specimens, and its potential application for fracturing coal roofs in coal mines has been proposed [8]. Guo et al. used soundless cracking agent in laboratory scale experiments to form a fracture network in shale specimens, in order to establish an evaluation method for the hydraulic fracturing in shale reservoirs [9]. More recently, Zhai et al. have proposed the application of non-explosive expansion method in the field of reservoir fracturing for the extraction of coal bed methane [10]. In the previous three cases, the acoustic emission (AE) technique was used for the dynamic monitoring and analysis of the fracture network formation. To the best of our knowledge, these are the only available studies where the effect of SCDAs in fracturing has been studied by means of the AE technique.

Although the optimum operation conditions of SCDAs, related to the external temperature, hole size and spacing, and mixing ratio of agent to water have been studied to some extent [2,11], the knowledge of the governing fracture generation and propagation mechanisms in concrete or rock masses is rather limited [5]. Regarding fracture mechanisms, quasi-static conditions can be considered in SCDA-induced rock fracturing, due to the observed stable crack propagation at low crack velocities, and micro-crack based sliding models are likely to be applied [5]. In this direction, the well-established monitoring technique of $\mathrm{AE}$ can provide valuable information on the fracture mechanisms in rocks and concrete structures under the effect of SCDAs [12-14].

The present study explores the potential use of the AE technique to study the SCDA-induced fracture process in concrete samples. The generation and propagation of micro-cracks into concrete specimens in unconfined state due to the addition of expansion mortar to pre-drilled holes is monitored for a period of 24-h, by an array of AE sensors. The experimental data are analyzed using parameter-based and statistical methodologies of acoustic emissions.

\section{EXPERIMENTAL SETUP}

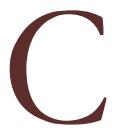

oncrete blocks used for pavement cover were cut in prismatic shape and a cylindrical hole, of $10 \mathrm{~mm}$ diameter and a depth of a few $\mathrm{cm}$, was drilled vertically to one of its surfaces (refer to Fig.1a). The pre-drilled hole was filled with DEXPAN soundless chemical demolition agent $[5,15]$, in the appropriate mixing ratio of water to cementitious powder ( 3.5:10), to achieve maximum expansive strenght (124 MPa, according to the manufacturer). The monitoring of concrete fragmentation in the absence of any confinement, by the AE technique was carried out at ambient pressure and temperature.

Seven AE piezoelectric sensors operated at 200-750 kHz (pico-sensors of Physical Acoustics Corporation) were mounted with silicon glue on all the surfaces of the concrete specimen. Pre-amplification of $40 \mathrm{~dB}$ was used in all sensors and the threshold of detection was set at $39 \mathrm{~dB}$, to avoid the background noise. The AE activity of the concrete block was monitored continously under the effect of the expansive mortar, for a period of 24 hours. A PCI-2 card based AE multichannel system (Physical Acoustics Corporation) was used to record the detected signals (hits) in each sensor and the AE hit parameters (amplitude, duration, rising time, rising angle, etc) were automatically extracted and further analysed by dedicated software (Aewin and NOESIS by Mistras Group). A characteristic recorded signal (hit) with the calculated based AE parameters is shown in Fig.2. Peak definition time (PDT), hit definition time (HDT) and hit lockout time (HLT) were set as 50,200 and $300 \mu \mathrm{s}$, respectively. The sampling rate was set at $5 \mathrm{Msamples} / \mathrm{s}$. The acoustic wave velocity of concrete specimens was determined experimentally by using two of the AE sensors in pulse-receive mode and found to be $(3120 \pm 70) \mathrm{m} / \mathrm{s}$. We have to mention that the highly inhomogeneous concrete specimen combined with the finite size of the AE sensors inevitably introduces errors in determining the location of the AE sources.

\section{RESULTS AND DISCUSSION}

\section{A parameter-based analysis of acoustic emissions}

he time history of the AE activity, expressed as the number of hits in time bins and their amplitudes, recorded in all channels during the effect of the cracking agent on the concrete block for a 24-h monitoring period, is illustrated in Fig.3, as a three-dimensional plot. From the recording time $t_{i} \approx 43618 \sec (\sim 12 \mathrm{~h})$ a large number of hits with ampli- 

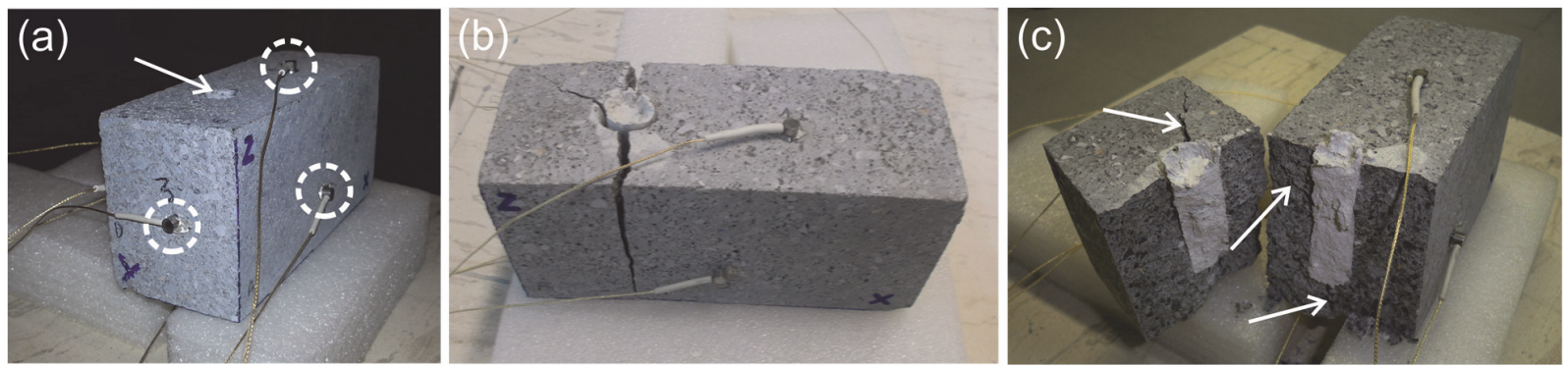

Figure 1: (a) The concrete specimen with the AE sensors mounted on it. The arrow indicates the pre-drilled hole filled with the expansive mortar and the dashed circles indicate the positions of the visible AE sensors; (b) The concrete block at the end of the monitoring where the fracture plane is parallel to the $\mathrm{y}-\mathrm{z}$ plane; (c) View of the two splitting pieces of the concrete block after its ultimate rupture. White arrows indicate main visible macro-cracks.

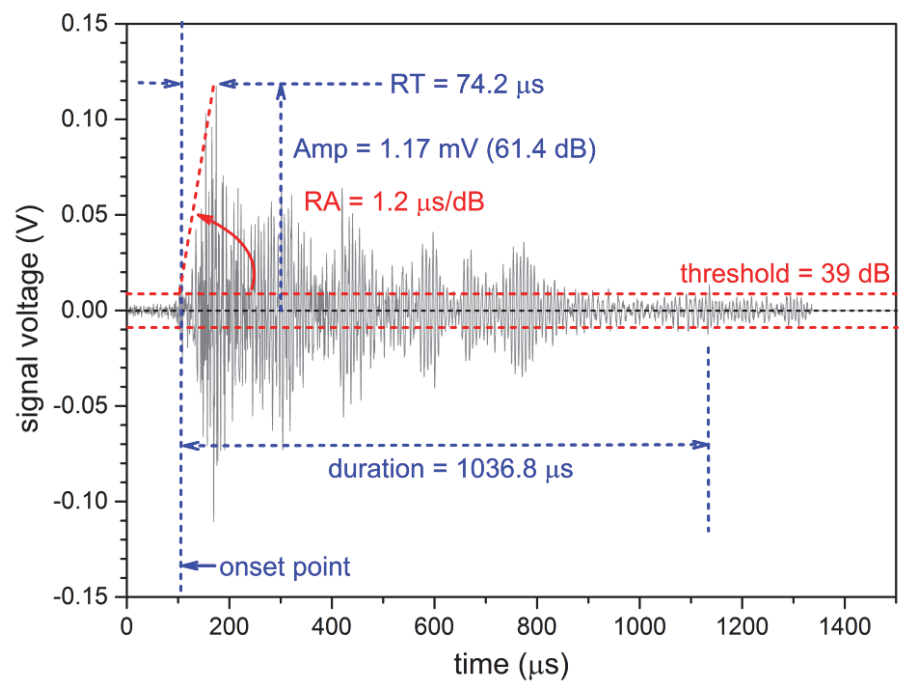

Figure 2: A representative recorded AE signal (hit) of moderate amplitude (61.4 dB), recorded in channel 6 , at relative recording time, $t=13675.24175475 \mathrm{~s}$. Rising angle $(\mathrm{RA}=1.2 \mu \mathrm{s} / \mathrm{dB})$ is defined as the ratio of rising time $(\mathrm{RT}=74.2 \mu \mathrm{s})$ to the corresponding amplitude.

tudes up to $90 \mathrm{~dB}$ starts to be observed, while afterwards, the recorded hits and their amplitudes are gradually decreased until the end of the monitoring test. This observed peak (refer to Fig.3) is attributed to the initiation of cracks generation into the concrete specimen, which occurs when the expansion stress of the cracking agent exceeds the tensile strength of the concrete specimen. The initiation of the fracturing process is manifested by a concentrated AE activity, that is, $3.5 \%$ (or 205 hits) of the total number of hits, over a short period of 0.5 sec. Subsequently, a considerable AE activity is recorded ( $\sim 45 \%$ of the total activity) that lasts for a long period of about $5.1 \mathrm{~h}$. This is related to the additional generation of micro-cracks and their coalescence which leads to the formation of the major macro-cracks in the concrete specimen, as it is shown in Fig.1(b, c). It should be noted here that the crack initiation was observed after $12 \mathrm{~h}$ which is far from the corresponding value suggested by the manufacturer, i.e. $2-8 \mathrm{~h}$ after the injection of SCDA. The latter, however, should be considered as the optimum value under certain operating conditions which are related to the ambient temperature, the water content and the diameter of the borehole.

A low AE activity is occasionally recorded in all channels before the observed onset of the fracturing process and from the beginning of the monitoring test, corresponding to $\sim 4 \%$ (i.e., 243 hits) of the total number of hits and to amplitudes $\leq 61 \mathrm{~dB}$. This activity should be associated with micro-cracks and friction effects, caused during the thermal expansion of the cracking agent within the pre-drilled hole of the concrete block. It is worth noting that there is no clear pre-failure activity, indicative of the upcoming fracturing process which started suddenly after about $12 \mathrm{~h}$ of the beginning of the monitoring test.

The high recorded AE activity that is related to the initiation of the cracking process in the concrete block is also reflected to the duration of the recorded hits in each of the 7 channels, as it is illustrated in Fig. 4 . According to Fig.4, the hits recorded around the time $t_{i} \approx 43618 \mathrm{sec}$ have duration as high as a few $\mathrm{ms}$, while afterwards and for the rest of the monitoring 


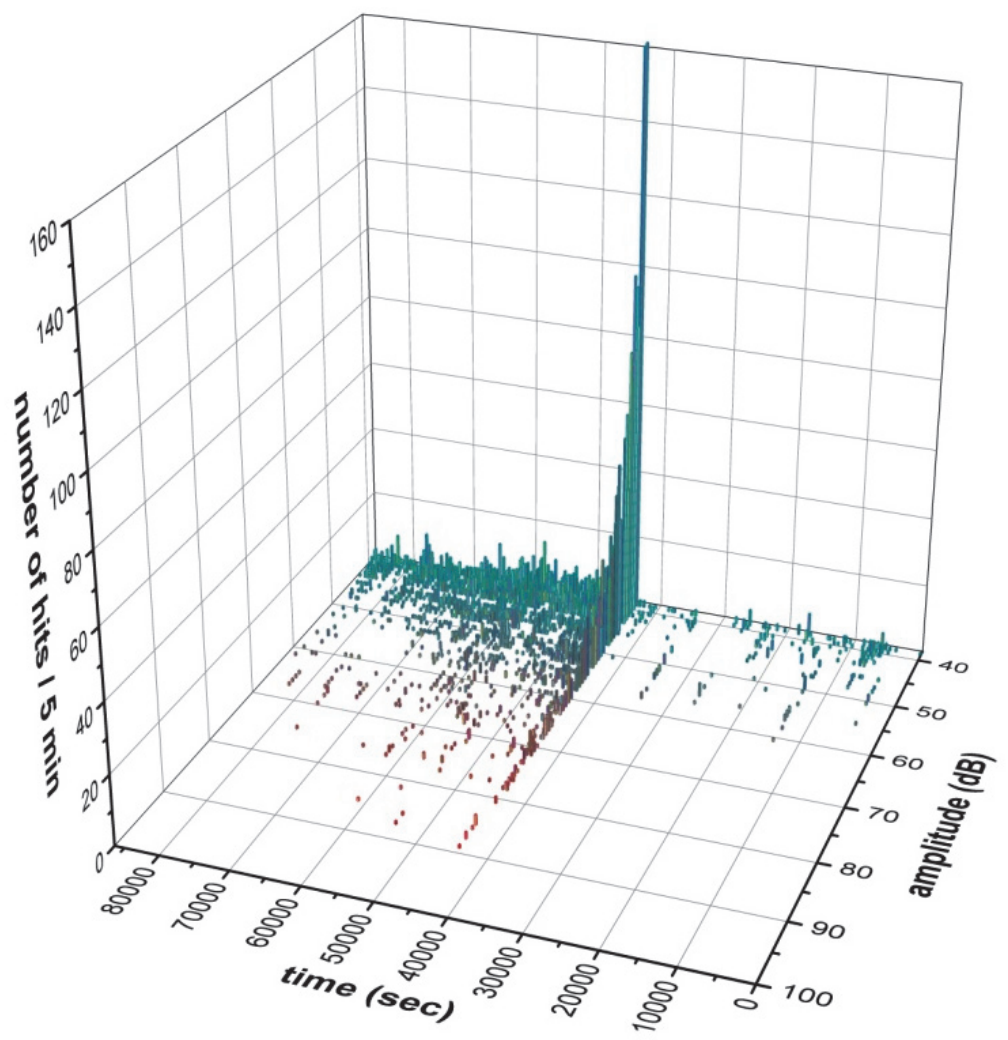

Figure 3: Time history of the distribution of amplitudes and the occurrence rate of the recorded AE hits in all channels, during the 24$\mathrm{h}$ monitoring of the fracturing process of the concrete specimen under the effect of the cracking agent.

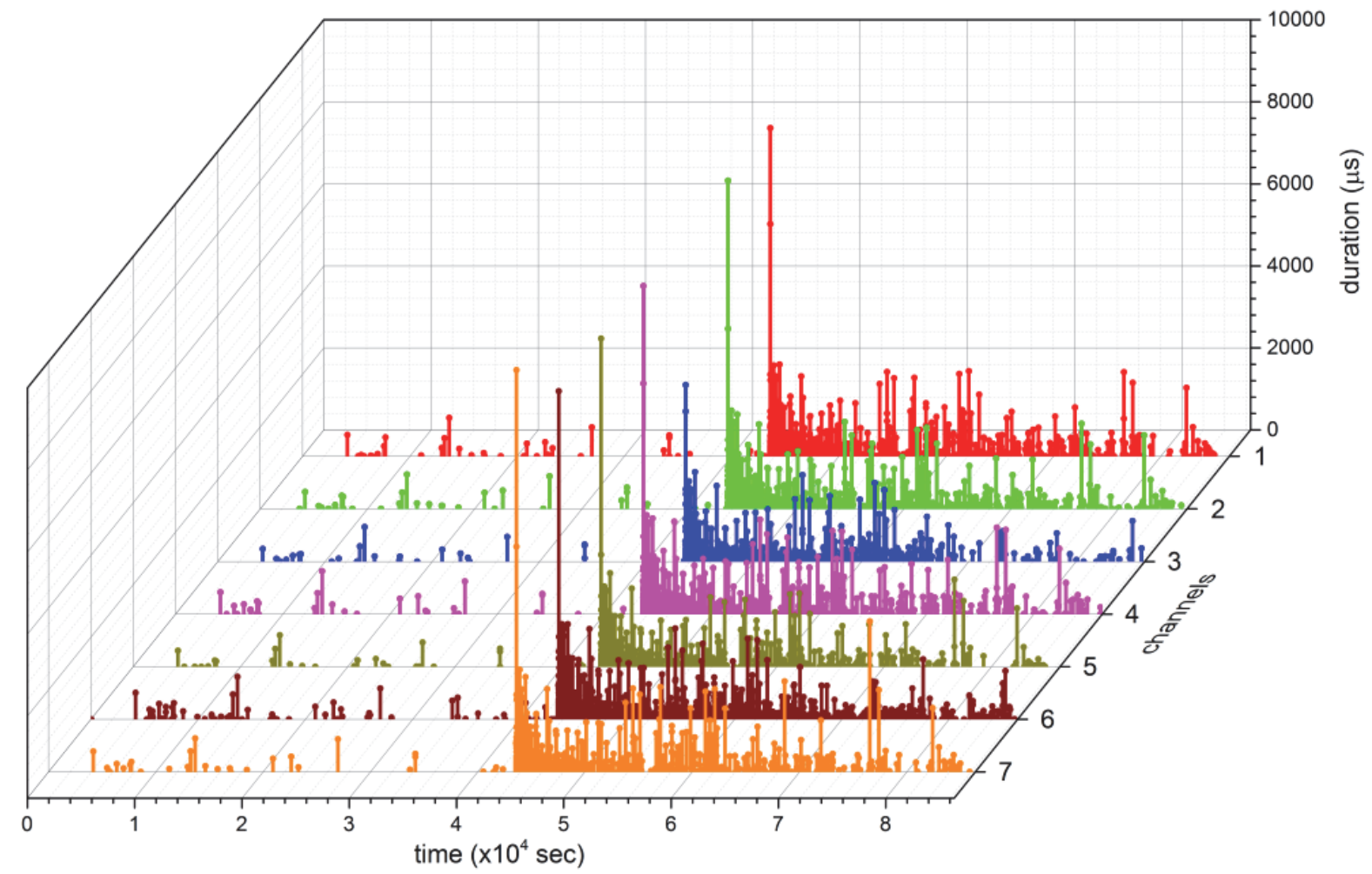

Figure 4: 3D plot of the time evolution of the duration (in $\mu \mathrm{s}$ ) of the hits in each recording channel, during the 24-h monitoring period of the concrete block subjected to the cracking agent charging. 


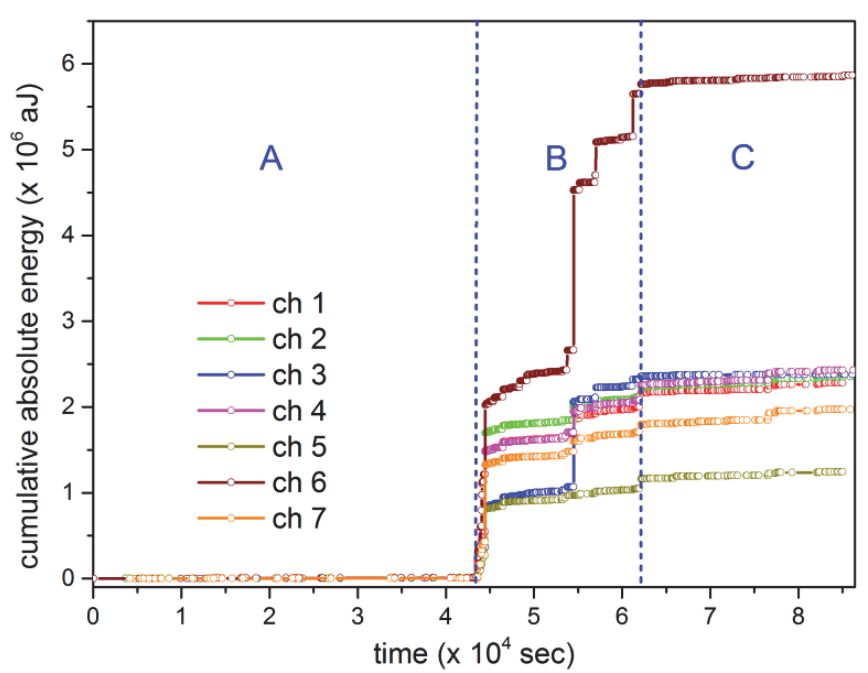

Figure 5: The cumulative absolute energy (in aJ) in each recorded channel, during the monitoring test of the concrete specimen. The overall monitoring period is divided into 3 stages, A, B and C (see text for details).

period, the recorded hits have substantially lower duration. Prior to the onset of the intense AE activity, the duration of the occasional recording hits are limited to less than $1 \mathrm{~ms}$, suggesting that the corresponding acoustic emissions have rather low energies and do not correspond to any significant precursory signal of the upcoming SCDA-induced cracking process.

Further information on the cracking process caused by the expansive agent in the concrete block can be derived from the time evolution of the cumulative absolute energy of the recorded hits in each channel, during the monitoring period (refer to Fig.5). The highest cumulative energy is recorded in channel 6, which is closer to the hole filled with the expansive agent. In all sensors, however, the recorded energy variations exhibit the same characteristics.

The overall monitoring period has been divided into 3 stages, depending on their specific characteristics. The "silent" stage A $(0-43618 \mathrm{sec})$ that appears prior to the initiation of the cracking process, corresponds to a limited number of hits $(\sim 4 \%$ of the total number) with low amplitudes and short durations and thus to a cumulative energy close to zero. In stage B which lasts for $5.1 \mathrm{~h}$, the cumulative energy of hits increases drastically exhibiting stepped-like behavior that corresponds to the formation of macro-cracks, as it is shown in Figs.1(b,c). This stage of strong AE activity can be considered as the effective period of action of the expansive agent in the fracture of the concrete specimen. Finally, in region C, although the recorded hits reach $20 \%$ of the total, the cumulative energy remains almost constant until the end of the monitoring test, due to the relatively short duration and the low amplitudes of the recorded hits. The separation of the monitoring period in the aforementioned stages, according to the values of the cumulative energy will be used later in the statistical analysis of the AE data.

The characterization of the cracking mode (tensile or mode-I and shear or mode-II) during the fracturing process of the concrete specimen can be achieved by correlating specific AE parameters, namely average frequency (AF) and rising angle (RA) [16-19]. This classification is based on the fact that the slower shear waves transmit more energy than the faster tensile waves, resulting to longer rising time and to a low frequency content of the recorded waveform, in opposition to the tensile waves which correspond to short rising times and higher frequencies [16].

The average frequency as a function of the rising angle of the recorded hits in channel 6 , for the stages A and B of the monitoring procedure is depicted in Fig.6a. Additionally, two representative recorded waveforms corresponding to different cracking modes and their frequency content are illustrated in Figs.6(b,c). We observe that the majority of the recorded hits are characterized by low values of RA and $\mathrm{AF}$ values in the low frequency range, i.e. $50-250 \mathrm{kHz}$. According to the aforementioned classification, these recorded hits are due to the formation of tensile cracks inside the concrete specimen. This is in accordance to the shape of the specimen after fracture, as it is depicted in Fig.1b. The compressive stresses that develop in the radial direction of the cylindrical hole and accumulate to the interface between the expansive agent and the walls, lead to indirect tensile stresses in the tangential direction of the hole which manifest as macro-cracks in the $y-z$ plane. The latter is the predominant plane of fracture, since the distance of the cylindrical hole from the side surfaces of the specimen is minimal in the y-direction, corresponding to minimal confinement from the surrounding material. These observations are in agreement with published results stating that SCDA-induced initial cracking occurs when the mean value of the tangential stress reaches the splitting tensile strength of the specimen and the crack propagation is caused mainly by mode-I type of failure $[5,11,20,21]$. 
As it is clearly observed in Fig.1c, additional smaller macro-cracks were also propagated from the interface of the hole with the concrete, without however reaching the outer surface, due to the higher surrounding confinement. These cracks should be of shear and mixed-type and they are observed in a smaller percentage, as it is evident in Fig.6a. Their presence in random directions could be attributed to the heterogeneity of the concrete specimen.

In both cases of tensile and shear cracks formed in the concrete specimen during the agent-induced fracturing, the different waveform features are clearly indicated in Figs.6(b,c). The low value of rise-time and the broad frequency content are indicative of tensile cracks (see Fig.6b). As for the shear cracks, rise-time is much longer and the frequency spectrum mainly contains low frequencies, as it is shown in Fig.6c.
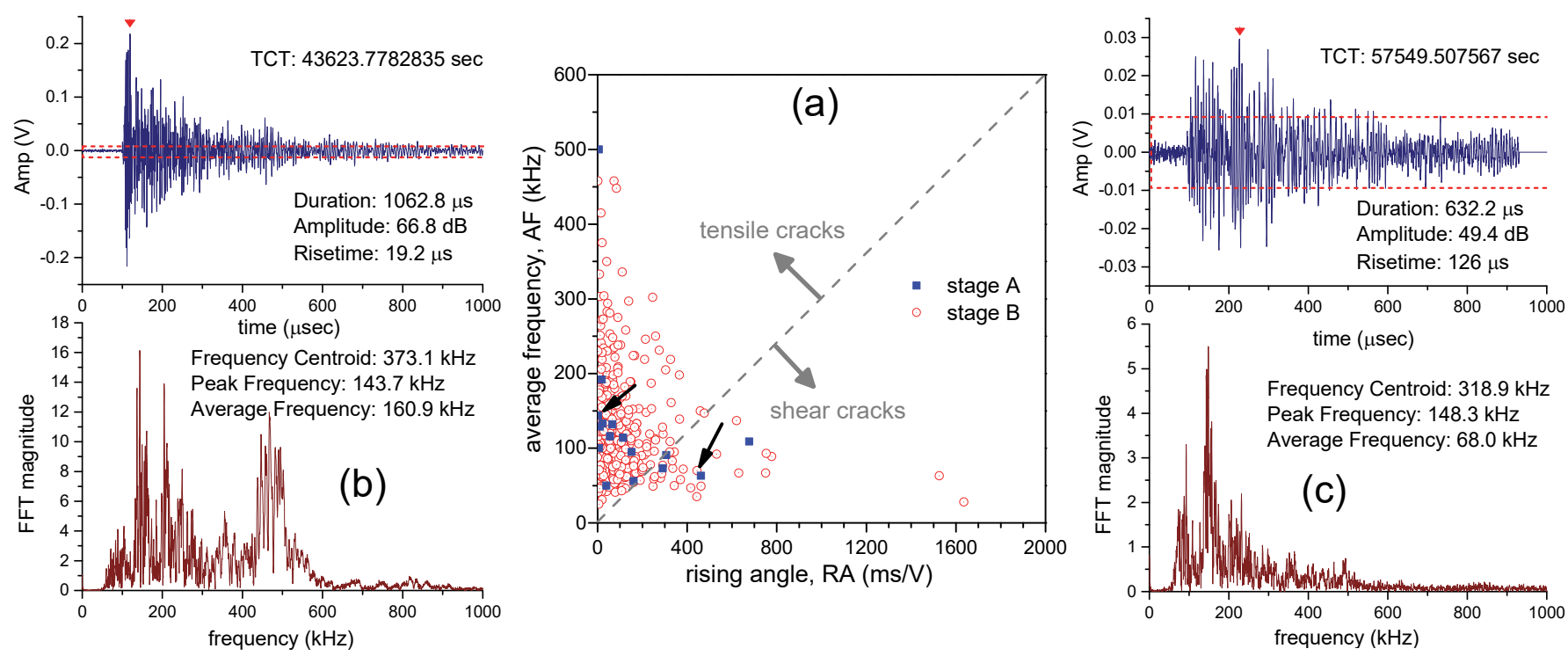

Figure 6: (a) Average frequency versus rising angle of the hits recorded in channel 6 with amplitudes greater than $45 \mathrm{~dB}$, during the two stages of the fracturing process (stages A and B) of the concrete specimen. The dashed grey line separates the two cracking modes; (b), (c) Two typical recorded waveforms [indicated by black arrows in (a)] attributed to tensile (b) and shear (c) cracking modes and their corresponding frequency spectra. The waveform characteristics are noted in each case. TCT stands for the threshold crossing time; red dashed lines indicate the threshold of detection $(39 \mathrm{~dB})$.

The use of 7 sensors in the monitoring test has enabled the determination of the location of AE sources. The principle of AE source localization is based on the minimization of the following quantity, chi square $\left(\chi^{2}\right)$, by using multiple regression analysis [22,23]:

$$
\chi^{2}=\sum_{i}\left(\Delta t_{i, o b s}-\Delta t_{i, c a l c}\right)^{2}
$$

where $\Delta t_{i, o b s}=t_{i}-t_{1}$ is the observed time difference calculated from the known arrival times in the first hit sensor and the ith sensor, and $\Delta t_{i, c a l c}$ is the calculated time difference, according to the following time-distance relationship

$$
\Delta t_{i, \text { calc }}=\left(\sqrt{\left(x_{i}-x_{s}\right)^{2}+\left(y_{i}-y_{s}\right)^{2}+\left(z_{i}-z_{s}\right)^{2}}-\sqrt{\left(x_{1}-x_{s}\right)^{2}+\left(y_{1}-y_{s}\right)^{2}+\left(z_{1}-z_{S}\right)^{2}}\right) / v
$$

In Eq.(2), $\left(x_{i}, y_{i}, z_{i}\right)$ and $\left(x_{s}, y_{s}, z_{s}\right)$ are the coordinates of the ith sensor and the unknown AE source location, respectively, in a $3 \mathrm{D}$ Cartesian coordinate system, and $v$ is the acoustic wave velocity in the material under test, which is considered isotropic and homogenous.

Three-dimensional location patterns of AE events at different selected times of the monitoring test are illustrated in Figs. $7(\mathrm{a}-\mathrm{d})$. The AE data were filtered to discard events with amplitudes lower than $45 \mathrm{~dB}$, in order to improve the overall location pattern. We observe that the locations of events before the initiation time of the fracturing process, i.e. in the range A, are rather concentrated at the bottom of the hole filled with the expansive mortar (refer to Fig.7a and Fig.5). During the evolution of the fracturing process (range B), new micro-cracks were formed and propagated around the hole, possibly at the interface of the concrete with the expansive mortar [refer to snapshots (b) and (c) of Fig.7]. These are reasonable findings, considering that the expansive pressure generated by the cracking agent is not uniformly distributed along 


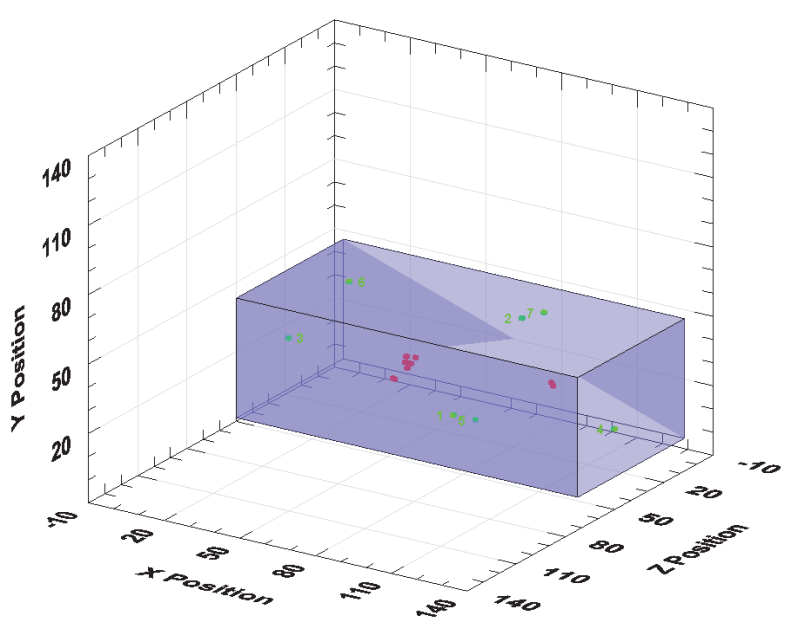

(a) recording time: 12:00:00 (43200 sec)

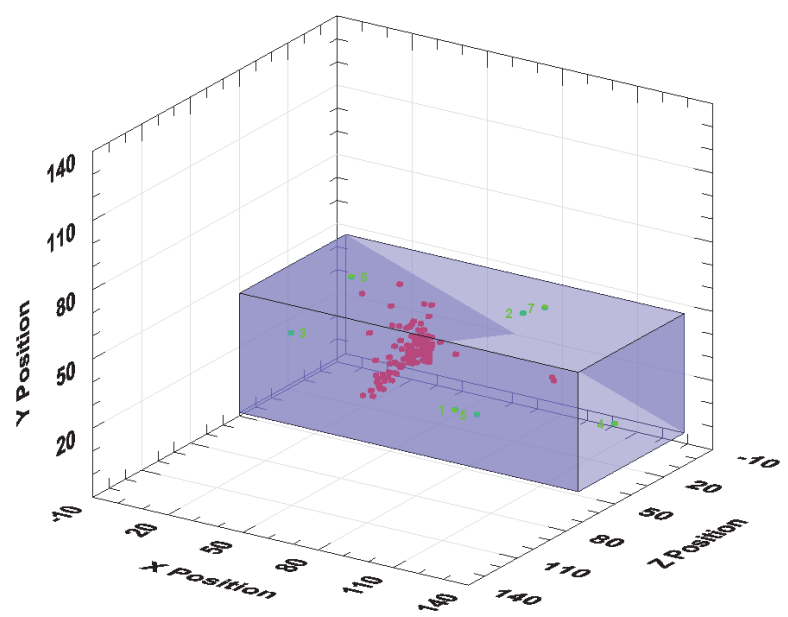

(c) recording time: 13:29:37 (48577 sec)

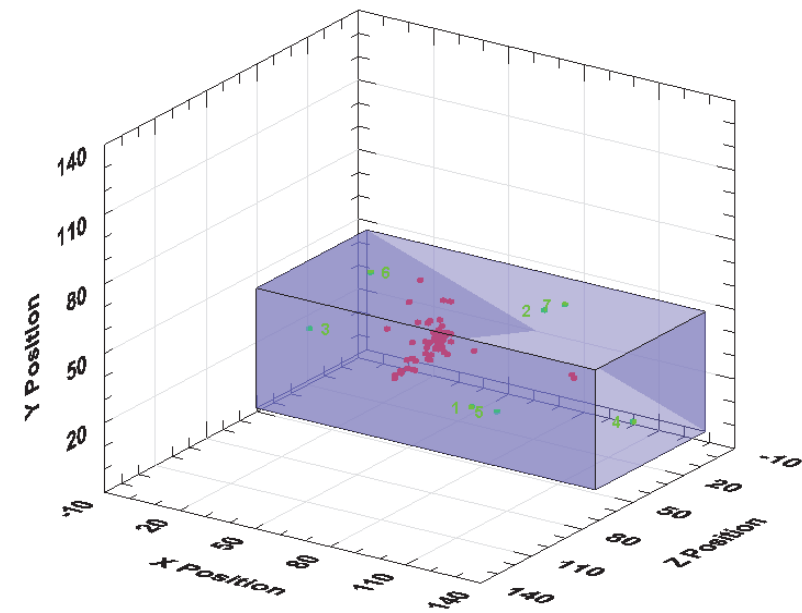

(b) recording time: $12: 11: 13(43873 \mathrm{sec})$

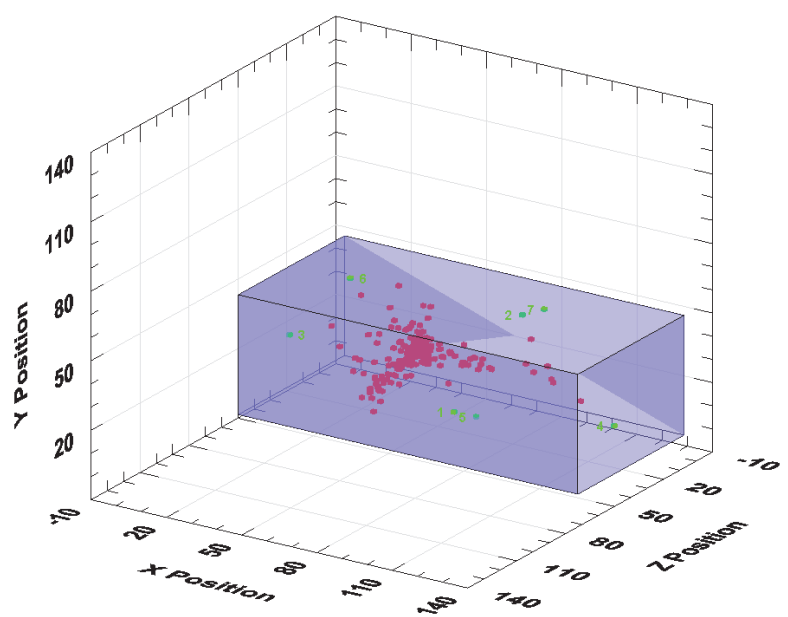

(d) recording time: 17:58:41 (64721 sec)

Figure 7: 3D location patterns of cumulative events at different selected times, during monitoring of the fracturing process in the concrete specimen. The numbered green dots indicate the positions of the $7 \mathrm{AE}$ sensors. The cylindrical hole was drilled along the z-direction (refer to Fig.1). Axes scale is in mm.

the cylindrical hole, due to its finite length and the absence of any confinement on the upper side of the hole. Thus, the expansive pressure should be higher in the inner part of the hole than at its edge. This results in the generation of microcracks starting from the inside and then extending towards the outside of the hole. As for the snapshot corresponding to the beginning of range C (Fig.7d), the distribution of the events has been further extended, due to the formation of macrocracks in the concrete specimen (Figs.1(b,c)).Further estimation of the events localization should be no longer valid, as the specimen has started to be divided into 2 pieces (refer to Fig.1b).

\section{Non-extensive statistical analysis of acoustic emissions}

The concept of non-extensive statistical physics (NESP) has been successfully applied to the investigation of complex systems in different length-scales, such as earthquakes and fracturing experiments in brittle materials [24-29]. NESP has been introduced by Tsallis in order to describe the non-equilibrium stationary states of a system which exhibits long-range interactions, memory effects and multifractality [30,31]. In this context, Tsallis proposed replacing the classical BoltzmanGibbs entropy with the generalized $q$-entropy (or Tsallis entropy) which is defined as:

$$
S_{q}=\frac{k_{B}}{q-1}\left(1-\int_{0}^{\infty}[p(X)]^{q} d X\right)
$$

where $k_{B}$ is Boltzmann's constant, $p(X)$ is the probability distribution function of the continuous variable $X$, and $q$ is a real parameter which describes the degree of non-extensivity of the system under consideration. For $q>1$ or $q<1$, the system 
has a sub-additive or super-additive behavior, respectively. If $q \rightarrow 1$, Tsallis entropy reduces to the classical BoltzmanGibbs entropy. In the case of the recorded AE activity during the fracture of brittle materials (concrete and rocks), the continuous variable $X$ corresponds to an $\mathrm{AE}$ parameter, such as the inter-event time or inter-event distance between two successive micro-cracks generated within the material, due to the applied mechanical stress [24,26,27].

The maximization of $q$-entropy under appropriate constraints generates probability distributions, the so-called $q$-distributions, such as $q$-exponential, $q$-Gaussian, $q$-Weibull distributions, etc [32-34]. The $q$-exponential distribution has been extensively used to describe the cumulative distribution functions (CDF) of seismic parameters in global and regional scale, and the AE activity in laboratory-scale fracturing experiments of rocks [24-28]. It is defined as follows

$$
P(>X)=\exp _{q}(-\beta X)=[1+(1-q) \beta X]^{1 /(1-q)}
$$

where the parameter $\beta$ is related to the Lagrange multiplier [27]. In the limit $q \rightarrow 1$, Eq.(4) leads to the ordinary exponential distribution.

In this study, NESP is applied to analyze the series of the inter-event times (IT, i.e. the time interval between successive micro-cracks) of the hits recorded during the SCDA-induced fracturing process of the concrete specimen (stage B). Specifically, we chose to use the sequence of the hits recorded to sensor with the maximum number of recorded hits, i.e., sensor 6 . The time history of the inter-event times $(\tau)$ of the recorded hits in channel 6 during the monitoring test, is shown in Fig.8a. The same data are also shown in a sequential order in Fig. $8 \mathrm{~b}$, to increase the resolution of observation during the fracture (stage $\mathrm{B}$ ), where most $\mathrm{AE}$ activity is recorded. We observe that $I T$ values cover more than 6 orders of magnitude, spanning from hundreds of seconds down to the millisecond range. In range A, ITs have very high values, because of the rare occurrence of hits. Instead, the initiation of the fracturing process (range B) causes an abrupt decrease of IT of more than 4 orders of magnitude (from $\sim 10^{2}$ to less than $10^{-2} \mathrm{sec}$ ), as it is clearly observed in Fig.8b. A subsequent characteristic decrease of $I T$ is observed at recording time $t \approx 43772 \mathrm{sec}$, i.e. after $154 \mathrm{sec}$ of the cracks' initiation (refer to Fig.8b)]. The prolonged recording of $I T$ with low to intermediate values $(\sim 0.1-10 \mathrm{sec})$ suggests that a quasi-static fracture is taking place during the effect of the cracking agent to the concrete specimen. This is consistent with published reports stating that the quasi-static conditions should be taken into account in fracture modelling of rocks with SCDAs [5].

An alternative approach for presenting the AE activity in terms of the inter-event times has been recently proposed by Triantis and Kourkoulis [35]. According to this methodology, a time function $F\left(\tau_{i}\right)$ is introduced, which represents the average frequency of occurrence of $\mathrm{AE}$ hits, in a sliding time window of $N$ consecutive hits with a mean value of the inter-event times, $\tau_{i}=\left(t_{N+i-1}-t_{i-1}\right) / N$. The time evolution of the function $F$ of the recorded hits in channel 6 for $N=10$, during the stage $\mathrm{B}$ of the fracture process is illustrated in Fig.8c. The observed peak of $F$ at 43618 sec is associated with the initiation of the fracture process which is significant during the first few minutes, but decreases afterwards. This behavior is quite similar to the observed fluctuations of the smoothed curve of $I T$, as it is shown in Fig. 8b. Depending on the low or higher values of $N$ which adjusts the time resolution of the evolution of the recorded hits during the test, the details of the AE activity can be revealed or shadowed, respectively. In the present case, for the optimum selected value of $N(N=10)$, the rate of AE hits as expressed by the function $F$ exhibit the same characteristics as those seen in Fig. 3 and Fig.8b.

Proceeding further with the analysis in the context of NESP, it should be noted that a single $q$-exponential function failed to fit the cumulative distribution of ITs in the entire region B. Thus, the aforementioned observed fluctuations of $I T$ in region $\mathrm{B}$ have prompted us to separate this region into 2 different sub-regions. The $1^{\text {st }}$ sub-region (B1), where $I T$ fluctuates considerably, includes hits from 71 to 470 and the $2^{\text {nd }}$ sub-region (B2) the remainder. In the latter case, the majority of ITs exhibit higher values on average. This separation of region B is also consistent with the approach mentioned above, regarding the values of the function $F$ in each sub-region [35]. In sub-region B1, the function $F$ maintains relatively high values, while in sub-region B2, F has low values, without any significant fluctuations.

The normalized cumulative distributions $(\mathrm{P}>\tau)$ of $I T$ associated to the recorded hits in channel 6 are depicted in Fig.9a,b, for the two distinct sub-regions of stage B, in a log-log representation. In Fig.9a, for low values of $I T(\tau<1$ sec $)$, the cumulative distribution was best fitted with a $q$-exponential function (red line), according to Eq.(4), while for higher values of $I T$, an ordinary exponential function (dashed green line) was sufficient to fit the data. Subsequently, the entire distribution of ITs in region B2 (hits 471-1340) is best fitted with a single $q$-exponential function, as it is depicted in Fig. 9b. In the latter case, the calculated $q$-value which is close to unity $(q=1.12 \pm 0.03)$ indicates a rather weak $q$-exponential behavior, suggesting that long-range interactions should be also weak.

On the contrary, the calculated high $q$-value $(q=2.58 \pm 0.05)$ in region B1 for low values of $I T$, suggests a highly sub-additive behavior, associated with a significantly well-organized fracturing process. However, the subsequent crossover to an exponential decay at higher $I T$ values is indicative of randomness in the fracturing process. Actually, the behavior of the CDFs of 
ITs in region B1 incorporates two different patterns, i.e. two regions of small and large values of IT that could be related to the micro-cracks generation and propagation in random positions in the interface between the SCDA and the surrounding concrete. Their subsequent coalescence in the macro-scale during the fracturing process resulting to ultimate failure, obeys a mechanism closer to a Boltzman-Gibbs statistics. A similar behavior of the $q$-index during three-point bending tests of cement mortar beams has been reported in ref.[36], where $q$-index was calculated in successive stages of the test, as the specimens approached failure, and was found to decrease, due to the different associated fracture mechanisms. More recently, NESP analysis was conducted at inter-event times of AE during repetitive loading/unloading cycles of cement mortar beams subjected to 3-point bending [37]. The experimental data were fitted by a $q$-exponential function; the entropic index $q$ was found to increase in each repeated cycle, approaching a critical value $(q=1.42)$ when the specimen reached ultimate failure.
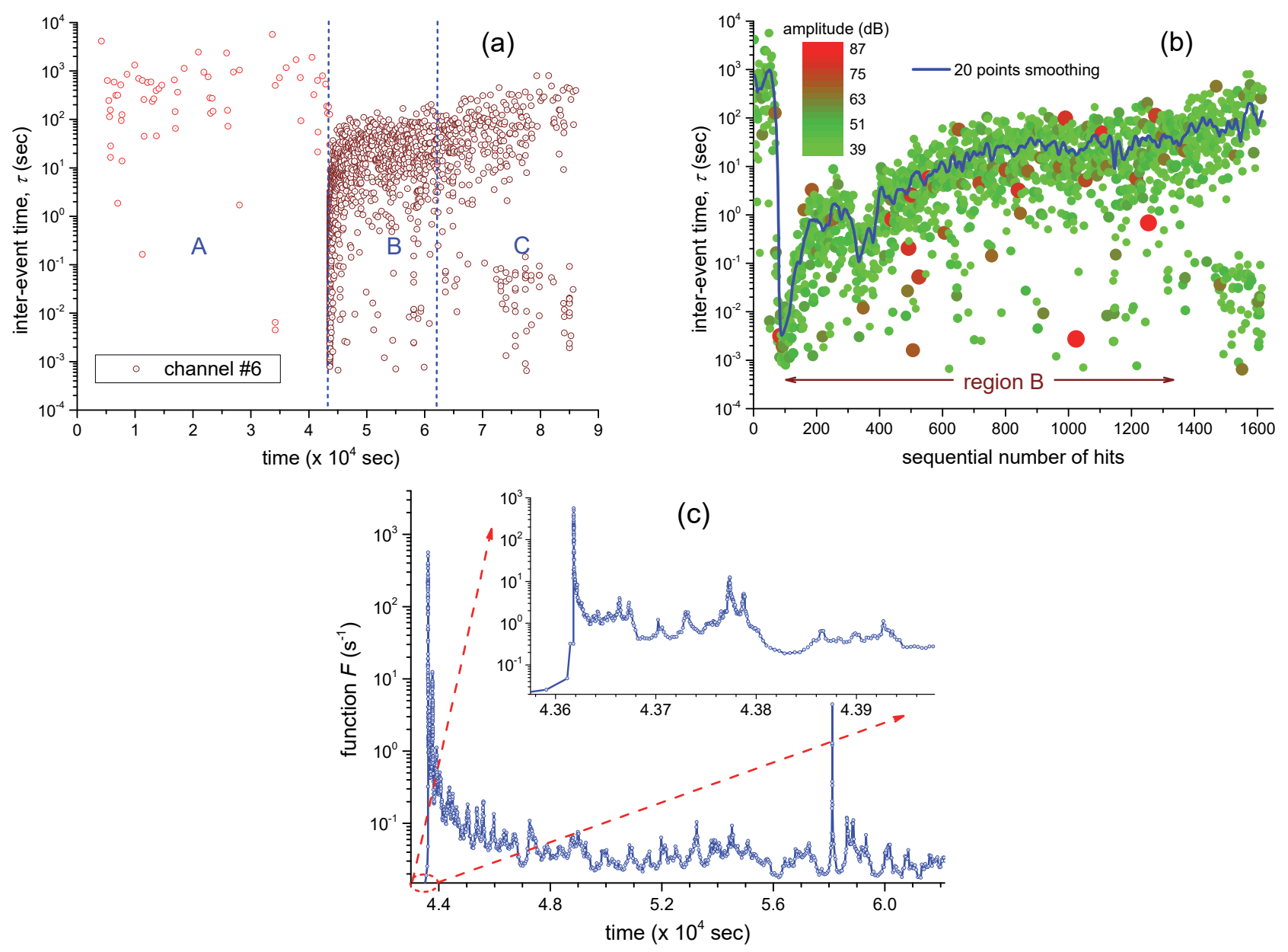

Figure 8: (a) Time evolution of the inter-event times of hits recorded to sensor with the maximum number of recorded hits (sensor 6) during the monitoring test of SCDA-induced concrete fracture. The 3 stages (A, B and C) refer to the separation applied in Fig.5, according to the variation of the cumulative absolute energy of the recorded hits. (b) The same data as in (a), in sequential order. The blue line represents a smoothed curve with an averaging method of 20 points. A color scale is used to distinguish the hits with different amplitudes. Only the data of stage B have been further analyzed in the context of NESP. (c) The function $F(\tau)$ of recorded hits in channel 6 , for the stage B $(43618-62145 \mathrm{~s})$ of the monitoring test. The number of consecutive hits is $N=10$. The inset shows in greater detail the first 6 minutes of the fracture process. Note the logarithmic scale in the vertical axis, in all cases.

\section{CONCLUSIONS}

$\mathrm{I}$ $\mathrm{n}$ the present work, the fracturing process of concrete specimens under the effect of a soundless chemical demolition agent was investigated by using the $\mathrm{AE}$ technique. Real-time monitoring of a concrete block with a pre-drilled hole filled with SCDA was performed for a period of $24 \mathrm{~h}$ by an array of $7 \mathrm{AE}$ sensors. The analysis of the experimental data 

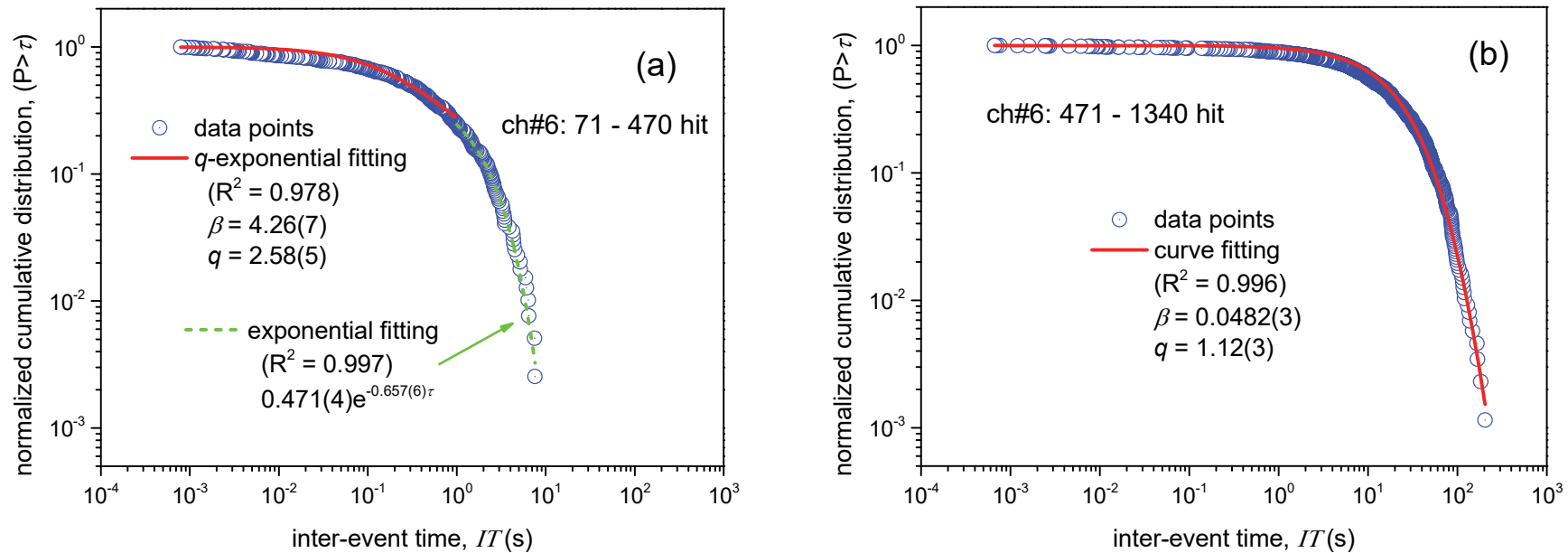

Figure 9: The normalized cumulative distribution of the inter-event times (blue circles) of hits recorded in channel 6, during the two substages of region B of the monitoring test. The fitting curves (red and dashed green lines) are also shown. Note the logarithmic scale in both axes. The fitting parameters are noted in each case.

reveals that fracture is suddenly observed after $12 \mathrm{~h}$, without any clear indication of pre-failure signals. The absolute energy and the inter-event times of the recorded hits have been used to distinguish the effective period of action of the cracking agent and the quasi-static behavior in the fracturing process, respectively. The correlation of the AE parameters (average frequency and rising angle) implies that the fracturing process is dominated by tensile cracks (mode-I type of fracture). The localization of the AE sources allows investigating the formation and propagation of micro-cracks around the pre-drilled hole. In this direction, we propose that the expansive agent placed into a hole of a solid material could be used as an artificial source of acoustic signals, in analogy to the lead pencil break tests (LPBT) for studying the propagation of elastic waves in materials. However, we have to point out that this proposed method, as a destructive test in contrast to the LPBT, cannot be used directly in the test specimens but should be applied separately to other suitably prepared specimens. Additionally, the use of the expansive agent may not be appropriate in the case of the high anisotropic rocks and should only be used in artificial materials. The statistical analysis of AE data in the frame of NESP provides evidence that the SCDA-induced fracture of concrete is a complex process where the long-range interactions of the micro-cracks should play a significant role to any possible model of the fracturing process.

The present investigation could be further extended to other brittle materials such as rocks and to complicated arrangement of holes that are used in practical applications, in order to monitor the evolution of the complex fracture network that will be developed under the effect of SCDAs. The influence of the essential parameters for the optimum usage of SCDAs, i.e. ambient temperature, water content, hole diameter and spacing between holes should be also investigated to reveal any possible correlations with the AE parameters.

\section{ACKNOWLEDGEMENTS}

e acknowledge support of this work by the project "HELPOS - Hellenic System for Lithosphere Monitoring, Greece" (MIS 5002697) which is implemented under the Action "Reinforcement of the Research and Innovation Infrastructure", funded by the Operational Programme "Competitiveness, Entrepreneurship and Innovation, Greece" (NSRF 2014-2020) and co-financed by Greece and the European Union (European Regional Development Fund).

\section{REFERENCES}

[1] Gambatese, J.A. (2003). Controlled Concrete Demolition Using Expansive Cracking Agents, J. Constr. Eng. Manag., 129(1), pp. 98-104, DOI: 10.1061/(ASCE)0733-9364(2003)129:1(98).

[2] Laefer, D.F., Ambrozevitch-Cooper, N., Huynh, M.P., Midgette, J., Ceribasi, S., Wortman, J. (2010). Expansive fracture agent behaviour for concrete cracking, Mag. Concr. Res., 62(6), pp. 443-452, DOI: 10.1680/macr.2010.62.6.443.

[3] Dessouki, A. El., Mitri, H. (2011). Rock Breakage Using Expansive Cement, Engineering, 03(02), pp. 168-173, DOI: $10.4236 /$ eng.2011.32020. 
[4] Hasanipanah, M., Jahed Armaghani, D., Monjezi, M., Shams, S. (2016). Risk assessment and prediction of rock fragmentation produced by blasting operation: a rock engineering system, Environ. Earth Sci., 75(9), pp. 1-12, DOI: $10.1007 /$ s12665-016-5503-y.

[5] De Silva, R.V., Gamage, R.P., Anne Perera, M.S. (2016). An alternative to conventional rock fragmentation methods using SCDA: A review, Energies, 9(11), pp. 1-31, DOI: 10.3390/en9110958.

[6] Guo, T., Zhang, S., Ge, H., Qu, Z. (2015). A Novel "Soundless Cracking Agent Fracturing” for Shale Gas Reservoir Stimulation, Int. J. Environ. Sci. Dev., 6(9), pp. 681-7, DOI: 10.7763/IJESD.2015.V6.680.

[7] Musunuri, A., Mitri, H. (2009). Laboratory investigation into rock fracturing with expansive cement, Int. J. Min. Miner. Eng., 1(4), DOI: 10.1504/IJMME.2009.029318.

[8] Xu, J., Zhai, C., Qin, L., Yu, G. (2017). Evaluation research of the fracturing capacity of non-explosive expansion material applied to coal-seam roof rock, Int. J. Rock Mech. Min. Sci., 94, pp. 103-111, DOI: $10.1016 /$ j.ijrmms.2017.03.004.

[9] Guo, T., Zhang, S., Ge, H., Wang, X., Lei, X., Xiao, B. (2015). A new method for evaluation of fracture network formation capacity of rock, Fuel, 140, pp. 778-787, DOI: 10.1016/j.fuel.2014.10.017.

[10] Zhai, C., Xu, J., Liu, S., Qin, L. (2018). Fracturing mechanism of coal-like rock specimens under the effect of nonexplosive expansion, Int. J. Rock Mech. Min. Sci., 103, pp. 145-154, DOI: 10.1016/j.ijrmms.2018.01.037.

[11] Natanzi, A.S., Laefer, D.F., Connolly, L. (2016). Cold and moderate ambient temperatures effects on expansive pressure development in soundless chemical demolition agents, Constr. Build. Mater., 110, pp. 117-127, DOI: 10.1016/j.conbuildmat.2016.02.016.

[12] Lockner, D. (1993). The role of acoustic emission in the study of rock fracture, Int. J. Rock Mech. Min. Sci. Geomech. Abstr., 30(7), pp. 883-899, DOI: 10.1016/0148-9062(93)90041-B.

[13] Shah, K.R., Labuz, J.F. (1995). Damage mechanisms in stressed rock from acoustic emission, J. Geophys. Res. Solid Earth, 100(B8), pp. 15527-15539, DOI: 10.1029/95JB01236.

[14] Aggelis, D.G., Soulioti, D. V., Sapouridis, N., Barkoula, N.M., Paipetis, A.S., Matikas, T.E. (2011). Acoustic emission characterization of the fracture process in fibre reinforced concrete, Constr. Build. Mater., 25(11), pp. 4126-4131, DOI: $10.1016 /$ j.conbuildmat.2011.04.049.

[15] https://www.dexpan.com/collections/dexpan-demolition-agent

[16] Aggelis, D.G. (2011). Classification of cracking mode in concrete by acoustic emission parameters, Mech. Res. Commun., 38(3), pp. 153-157, DOI: 10.1016/j.mechrescom.2011.03.007.

[17] Aggelis, D.G., Mpalaskas, A.C., Matikas, T.E. (2013). Investigation of different fracture modes in cement-based materials by acoustic emission, Cem. Concr. Res., 48, pp. 1-8, DOI: 10.1016/j.cemconres.2013.02.002.

[18] Farhidzadeh, A., Mpalaskas, A.C., Matikas, T.E., Farhidzadeh, H., Aggelis, D.G. (2014). Fracture mode identification in cementitious materials using supervised pattern recognition of acoustic emission features, Constr. Build. Mater., 67(PART B), pp. 129-138, DOI: 10.1016/j.conbuildmat.2014.05.015.

[19] Tsangouri, E., Aggelis, D., Matikas, T., Mpalaskas, A. (2015). Acoustic Emission Activity for Characterizing Fracture of Marble under Bending, Appl. Sci., 6(1), pp. 6, DOI: 10.3390/app6010006.

[20] Harada T., Idemitsu T., Watanabe A., Takayama S. (1989). The Design Method for the Demolition of Concrete with Expansive Demolition Agents. In: Shah S.P., Swartz S.E. (eds) Fracture of Concrete and Rock. Springer, New York.

[21] Shang, J., Zhao, Z., Aliyu, M.M. (2018). Stresses induced by a demolition agent in non-explosive rock fracturing, Int. J. Rock Mech. Min. Sci., 107(December), pp. 172-180, DOI: 10.1016/j.ijrmms.2018.04.049.

[22] Agioutantis, Z., Kaklis, K., Mavrigiannakis, S., Verigakis, M., Vallianatos, F., Saltas, V. (2016). Potential of acoustic emissions from three point bending tests as rock failure precursors, Int. J. Min. Sci. Technol., 26(1), pp. 155-160, DOI: $10.1016 /$ j.ijmst.2015.11.024.

[23] Kaklis, K., Mavrigiannakis, S., Saltas, V., Vallianatos, F., Agioutantis, Z. (2017). Using acoustic emissions to enhance fracture toughness calculations for CCNBD marble specimens, Frat. Ed Integrita Strutt., 11(40), DOI: $10.3221 /$ IGF-ESIS.40.01.

[24] Vallianatos, F., Benson, P., Meredith, P., Sammonds, P. (2012). Experimental evidence of a non-extensive statistical physics behaviour of fracture in triaxially deformed Etna basalt using acoustic emissions, Epl, 97(5), DOI: $10.1209 /$ 0295-5075/97/58002.

[25] Vallianatos, F., Sammonds, P. (2013). Evidence of non-extensive statistical physics of the lithospheric instability approaching the 2004 Sumatran-Andaman and 2011 Honshu mega-earthquakes, Tectonophysics, 590, pp. 52-58, DOI: $10.1016 /$ j.tecto.2013.01.009.

[26] Vallianatos, F., Papadakis, G., Michas, G. (2016). Generalized statistical mechanics approaches to earthquakes and tectonics, Proc. R. Soc. A Math. Phys. Eng. Sci., 472(2196), DOI: 10.1098/rspa.2016.0497. 
[27] Saltas, V., Vallianatos, F., Triantis, D., Stavrakas, I. (2018). Complexity in Laboratory Seismology: From Electrical and Acoustic Emissions to Fracture, in: T. Chelidze, L. Telesca, F. Vallianatos (Eds.), Complexity of Seismic Time Series: Measurement and Application, 1st ed., Elsevier, pp. 239-273, ISBN: 978-0-12-813138-1.

[28] Chochlaki, K., Vallianatos, F., Michas, G. (2018). Global regionalized seismicity in view of Non-Extensive Statistical Physics, Phys. A Stat. Mech. Its Appl., 493, pp. 276-285, DOI: 10.1016/j.physa.2017.10.020.

[29] Michas, G., Vallianatos, F. (2018). Stochastic modeling of nonstationary earthquake time series with long-term clustering effects, Phys. Rev. E, 98(4), pp. 042107, DOI: 10.1103/PhysRevE.98.042107.

[30] Tsallis, C. (1988). Possible generalization of Boltzmann-Gibbs statistics, J. Stat. Phys., 52(1-2), pp. 479-87, DOI: $10.1007 /$ BF01016429.

[31] Tsallis, C. (2009). Nonadditive entropy and nonextensive statistical mechanics -an overview after 20 years, Brazilian J. Phys., 39(2a), pp. 337-356, DOI: 10.1590/S0103-97332009000400002.

[32] Picoli, S, Jr, Mendes, R.S., Malarne, L.C., Santos, R.P.B. (2009). Q-Distributions in Complex Systems: a Brief Review, Braz J Phys, 39(2A), pp. 468-474, DOI: 10.1590/S0103-97332009000400023.

[33] Vallianatos, F., Sammonds, P. (2010). Is plate tectonics a case of non-extensive thermodynamics?, Phys. A Stat. Mech. Its Appl., 389(21), pp. 4989-4993, DOI: 10.1016/j.physa.2010.06.056.

[34] Vallianatos, F. (2018). A non-extensive statistical mechanics view on easter island seamounts volume distribution, Geosci., 8(2), DOI: 10.3390/geosciences8020052.

[35] Triantis, D., Kourkoulis, S.K. (2018). An alternative approach for representing the data provided by the Acoustic Emission technique, Rock Mech. Rock Eng., 51(8), pp. 2433-2438, DOI: 10.1007/s00603-018-1494-1.

[36] Stergiopoulos, C., Stavrakas, I., Hloupis, G., Triantis, D., Vallianatos, F. (2013). Electrical and Acoustic Emissions in cement mortar beams subjected to mechanical loading up to fracture, Eng. Fail. Anal., 35, pp. 454-461, DOI: $10.1016 /$ j.engfailanal.2013.04.015.

[37] Stavrakas, I., Triantis, D., Kourkoulis, S.K., Pasiou, E.D., Dakanali, I. (2016). Acoustic emission analysis of cement mortar specimens during three point bending tests, Lat. Am. J. Solids Struct., 13(12), pp. 2283-2297, DOI: $10.1590 / 1679-78252486$. 